\title{
Convex hull and tour crossings in the Euclidean traveling salesperson problem: Implications for human performance studies
}

\author{
IRIS VAN ROOIJ, ULRIKE STEGE, and ALISSA SCHACTMAN \\ University of Victoria, Victoria, British Columbia, Canada
}

\begin{abstract}
Recently there has been growing interest among psychologists in human performance on the Euclidean traveling salesperson problem (E-TSP). A debate has been initiated on what strategy people use in solving visually presented E-TSP instances. The most prominent hypothesis is the convex-hull hypothesis, originally proposed by MacGregor and Ormerod (1996). We argue that, in the literature so far, there is no evidence for this hypothesis. Alternatively we propose and motivate the hypothesis that people aim at avoiding crossings.
\end{abstract}

The traveling salesperson problem (TSP; traditionally referred to as the traveling salesman problem) can be informally described as follows. For a given set of points and costs between each pair of points, find a cheapest tour that visits each point exactly once. In the Euclidean version of the traveling salesperson problem (E-TSP), the points are positioned in the Euclidean plane and the costs are the distances implied by the Euclidean geometry. The study presented by MacGregor and Ormerod (1996) is one of the first psychological studies that explicitly and primarily focuses on human performance on E-TSP (but see also Polivanova, 1974). Their study has motivated further psychological research on the topic and has initiated a debate about what strategy people use in solving visually presented E-TSP instances ${ }^{1}$ (Graham, Joshi, \& Pizlo, 2000; MacGregor, Ormerod, \& Chronicle, 1999, 2000; Ormerod \& Chronicle, 1999; Vickers, Butavicius, Lee, \& Medvedev, 2001; see also Lee \& Vickers, 2000, for a commentary on MacGregor \& Ormerod, 1996, and see MacGregor \& Ormerod, 2000, for a reply).

MacGregor and Ormerod (1996) proposed the convexhull hypothesis, which states that people use the convex hull as part of their strategy to construct E-TSP tours. The convex hull of a set of points in the plane is the smallest convex polygon that encloses all the points in the set (the points on the convex hull are also called boundarypoints and the remaining points are then called

This research was supported by a research grant from the National Science and Engineering Research Council (NSERC) of Canada and a research grant from the University of Victoria, both awarded to U.S. While working on this article, I.R. was additionally supported by NSERC Grant OGP0121280-97, awarded to Helena Kadlec. We thank Jim MacGregor, Tom Ormerod, Ed Chronicle, Douglas Vickers, Marcus Butavicius, Michael Lee, and Andrei Medvedev for sharing their data. Special thanks to Jim MacGregor, Evan Heit, Tom Ormerod, Michael Lee, and an anonymous reviewer for their helpful comments and suggestions. Correspondence should be addressed to I. van Rooij, Department of Psychology, P.O. Box 3050, University of Victoria, Victoria, BC, V8W 3P5 Canada (e-mail: irisvr@uvic.ca). interior points; see Figure 1). The convex-hull hypothesis is based on the assumptions that (1) people can identify the convex hull of a point set in the plane via an automatic perceptual process, and (2) people are sensitive to the principle that optimal tours connect points on the convex hull in order of adjacency (see also MacGregor et al., 1999, 2000; Ormerod \& Chronicle, 1999).

The purpose of this commentary is to point out the absence of evidence for the convex-hull hypothesis in the literature on human performance on E-TSP. We further suggest an alternative hypothesis, the crossing-avoidance hypothesis.

\section{A CROSSING-FREE TOUR THAT RESPECTS THE CONVEX HULL IS NO SURPRISE}

MacGregor and Ormerod (1996) found that tours proposed by human participants tend to connect convex-hull points in order, and claimed that this finding provides evidence for the convex-hull hypothesis (see also Graham et al., 2000; MacGregor et al., 1999, 2000; Ormerod \& Chronicle, 1999). We show that, because tours produced by participants seldom contain crossings, this conclusion is not warranted.

We start by stating two observations.

Observation la. A tour that does not follow the convex hull contains at least one crossing.

For an illustration of a proof refer to Figure 1. Note that Observation $1 \mathrm{a}$ is equivalent to Observation $1 \mathrm{~b}$.

Observation $1 b$. A tour that does not contain any crossings follows the convex hull.

Observation 2. There exist tours with at least one crossing that follow the convex hull.

Consistent with Observations 1 and 2, MacGregor and Ormerod (1996, pp. 531-532) wrote: "Failure to connect 


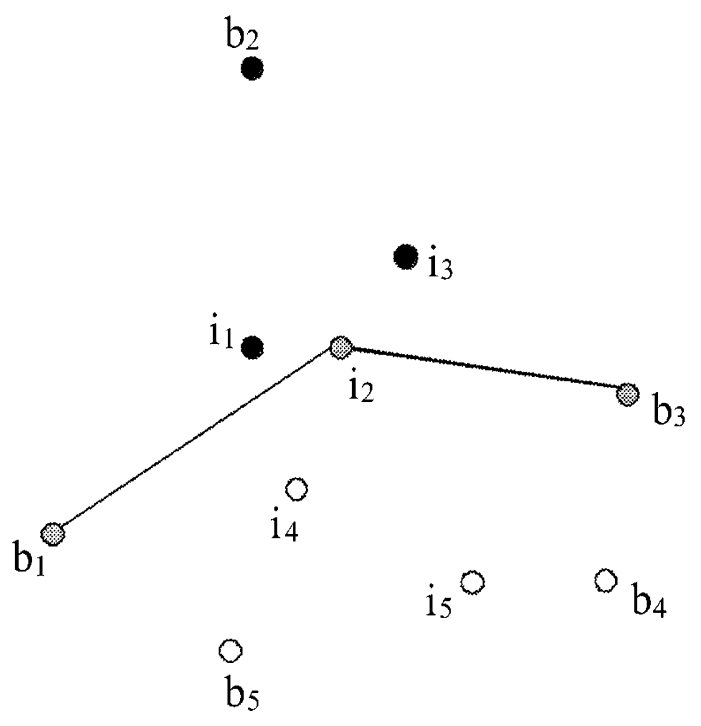

Figure 1. An illustration of what happens when convex-hull points are not connected in order of adjacency. Given a set of points $P$, with boundary points $b_{1}, b_{2}, \ldots, b_{5}$ and interior points $i_{1}$, $i_{1}, \ldots, i_{5}$. In this example, a path is created from boundary point $b_{1}$, via interior point $i_{2}$, to boundary point $b_{3}$, without visiting boundary point $b_{2}$ between $b_{1}$ and $b_{3}$. Note that this path divides the set of points in two subsets $\boldsymbol{P}_{\mathbf{1}}$ (white + gray points) and $P_{2}$ (black + gray points), with $P_{1} \cap P_{2}=\left\{b_{1}, i_{2}, b_{3}\right\}$ (gray points). Since the tour has to visit all the points in $P$, to complete the tour at least one edge must eventually cross the path from $b_{1}$ to $i_{2}$, to $b_{3}$, in order to come from $P_{1}$ to $P_{2}$, or vice versa.

boundary points in order of adjacency automatically creates a solution with crossed arcs. However, crossed arcs can also occur when boundary points are connected in order." Inconsistent with Observation 1, they further reported that "almost invariably, subjects connected boundary points in order of adjacency and, equally invariably produced no crossed arcs" (p. 536). This last sentence seems to state two independent findings. In a similar vein, MacGregor et al. (2000, p. 1184) enumerated the main findings of MacGregor and Ormerod (1996), with one point being that "people produce paths that connect boundary points in sequential order of adjacency (449 of 455 solutions adhered to this principle)," and another, again seemingly independent point being that "few solutions have lines that cross (11 of the 455 solutions)." Note, however, that the fact that 444 of the proposed tours contain no crossing logically implies that these 444 tours visit the boundary points in order of adjacency (Observation 1 ). Therefore the finding that the majority of the subjects connected boundary points in order of adjacency is no surprise, but a logical consequence of the fact that the majority of tours have no crossings. In other words, these two findings are not independent empirical findings.

\section{DO TOURS WITH CROSSINGS FOLLOW THE CONVEX HULL?}

Observation 1 implies that we have to consider tours with crossings to provide evidence for the hypothesis that people aim at following the convex hull (Table 1). Table 2 gives an overview of the number and proportion of tours with crossings 2 that follow the convex hull, for Experiments 1 and 2 of MacGregor and Ormerod (1996), Experiments 1, 2, and 3 of MacGregor et al. (1999), the optimization group (Group O) in Experiment 1 of Vickers et al. (2001), and the adult group in the experiment by Schactman (2002). ${ }^{3}$ For ease of presentation, the data in Table 2 are collapsed over instances and participants within each experiment. We note that the occurrence of crossings does not seem to be strictly instance specific, nor does the failure to avoid crossings seem to be strictly participant specific. Namely, for 3 of the 6 instances in Experiment 1, for 4 of the 7 instances in Experiment 2 of MacGregor and Ormerod (1996), for 5 of the 6 instances of Vickers et al. (2001), and for 8 of the 15 instances of Schactman (2002), at least one tour with crossings was observed. The tours with crossings in Experiments 1 and 2 in MacGregor and Ormerod (1996) were produced by 5 and 1 participants, respectively, in Vickers et al. (2001) by 7 participants, and in Schactman (2002) by 4 participants.

Table 2 shows that overall about $38 \%$ of the tours with crossings visited the convex-hull points in order of adjacency. From Table 2 we can conclude that there is no evidence of a tendency to follow the convex hull.

\section{IS THERE EVIDENCE FOR THE CONVEX- HULL HYPOTHESIS IN THE LITERATURE?}

In the literature seven findings are presented as evidence for the convex-hull hypothesis (MacGregor \& Ormerod, 1996; MacGregor et al., 1999, 2000): (1) People tend to follow the convex hull, (2) response uncertainty is a function of the number of interior points, (3) people tend to produce tours without crossings, (4) people tend to produce tours with relatively few indentations (an indentation in a tour occurs if at least one interior point is visited between two boundary points), (5) performance is better when interior points are located relatively close to the convex hull than when they are located far away from the convex hull, (6) tours produced by a convexhull heuristic (see MacGregor et al., 2000) are close in length to tours produced by humans, and (7) this heuristic's performance is qualitatively similar to human performance. We have argued above that Finding 1 does not

Table 1

The Three Conjunctions of Findings for Tour Crossings (Yes/No) and Tours Following the Convex Hull (Yes/No) That Are Possible

\begin{tabular}{|c|c|c|c|}
\hline $\begin{array}{l}\text { Tour Has } \\
\text { Crossings }\end{array}$ & $\begin{array}{l}\text { Tour Follows } \\
\text { Convex Hull }\end{array}$ & $\begin{array}{c}\text { Convex-Hull } \\
\text { Hypothesis }\end{array}$ & $\begin{array}{c}\text { Crossing-Avoidance } \\
\text { Hypothesis }\end{array}$ \\
\hline Yes & Yes & + & - \\
\hline Yes & No & - & - \\
\hline No & Yes & $\mathrm{n}$ & + \\
\hline
\end{tabular}

Note-For each conjunction it is indicated whether it constitutes evidence for $(+)$, evidence against $(-)$, or evidence neither for nor against (n) the convex-hull hypothesis and/or the crossing-avoidance hypothesis. 
Table 2

Descriptives for Tours With Crossings From Previous Research

\begin{tabular}{|c|c|c|c|c|c|c|}
\hline \multirow[b]{2}{*}{ Study } & \multirow{2}{*}{$\begin{array}{l}\text { Total No. } \\
\text { Instances }\end{array}$} & \multicolumn{2}{|c|}{$\begin{array}{c}\text { No. Tours } \\
\text { With Crossings } \\
\end{array}$} & \multicolumn{2}{|c|}{$\begin{array}{c}\text { No. Tours } \\
\text { With Crossings } \\
\text { That Follow } \\
\text { Convex Hull }\end{array}$} & \multirow{2}{*}{$\begin{array}{l}\text { No. Tours } \\
\text { With } \\
\text { Nonphysical } \\
\text { Crossings }\end{array}$} \\
\hline & & No. & Prop. & No. & Prop. & \\
\hline \multicolumn{7}{|l|}{ MacGregor \& Ormerod (1996) } \\
\hline Experiment 1 & 315 & 5 & 0.02 & 0 & 0.00 & 0 \\
\hline Experiment 2 & 140 & 6 & 0.04 & 5 & 0.83 & 0 \\
\hline \multicolumn{7}{|l|}{ MacGregor et al. (1999) } \\
\hline Experiment 1 & 103 & 20 & 0.19 & 6 & 0.30 & 11 \\
\hline Experiment 2 & 34 & 2 & 0.06 & 1 & 0.50 & 0 \\
\hline Experiment 3 & 856 & 65 & 0.08 & 23 & 0.35 & 4 \\
\hline \multicolumn{7}{|l|}{ Vickers et al. (2001) } \\
\hline Experiment 1 (Group O) & 108 & 9 & 0.08 & 3 & 0.33 & 2 \\
\hline \multicolumn{7}{|l|}{ Schactman (2002) } \\
\hline Adult group & 270 & 10 & 0.04 & 7 & 0.70 & 0 \\
\hline Total & 1,826 & 117 & 0.06 & 45 & 0.38 & 16 \\
\hline
\end{tabular}

Note-The process by which stimuli were generated was not homogeneous across studies. In Experiments 1 and 2 of MacGregor and Ormerod (1996) and in Experiment 3 of MacGregor et al. (1999), the process can be characterized as random with strong constraints, in Experiment 2 of MacGregor et al. (1999) as nonrandom, and in Experiment 1 of MacGregor et al. (1999), Vickers et al. (2001), and Schactman (2002) as random.

provide evidence for the convex-hull hypothesis. In the following we argue that this also holds for Findings 2-7.

As MacGregor and Ormerod argued (1996, p. 528), if the tour is constrained to follow the convex hull, then decreasing the number of interior points (while keeping the total number of points fixed) reduces the degrees of freedom for connecting the points. We have shown that the absence of crossings implies a tour that follows the convex hull. Thus, Finding 2 is also a consequence of Finding 3. Finding 3 does not provide evidence for the convexhull hypothesis, simply because following the convex-hull points in order of adjacency does not prevent crossings (Observation 2). As pointed out by MacGregor and Ormerod (1996; see also Lee \& Vickers, 2000) and MacGregor et al. (2000), given the highly constrained nature of the stimuli used, Findings 4 and 5 may be artifacts. Because the optimal tours for the instances used by MacGregor and Ormerod (1996) all had very few indentations (see, e.g., Figure 1 in Ormerod \& Chronicle, 1999), Finding 4 follows directly from the close to optimal performance by participants on these instances. We further note that Finding 4 is unlikely to be replicated with random instances, since connecting boundary points to each other is in general clearly a nonoptimal strategy. To argue that Finding 6 does not provide evidence for the convex-hull hypothesis, we note that human performance in the considered experiments is close to optimal. Any heuristic that models human performance will have to produce close to optimal tours (and thus close to human performance). Thus comparing only lengths of tours produced by humans and heuristics is not informative about the strategy of humans. We studied the convex-hull heuristic (cheapest insertion criterion) described by MacGregor et al. (2000, pp. 1184-1186) and disagree that it qualitatively models human behavior (Finding 7). MacGregor et al. (2000) distinguished be- tween sketched and connected arcs. The heuristic allows the construction of a closed path consisting of connected arcs only, even before all interior points are included in the tour (see Figure 2 for an illustration). As a consequence, it can happen that the heuristic visits certain points (and arcs) several times before the tour is completed. Also, previously connected arcs may need to be disconnected and replaced by a subpath in order to complete the tour. This behavior of the heuristic is inconsistent with the motivation of MacGregor et al. (2000) to model the sequential character of tour construction by humans.

\section{THE CROSSING-AVOIDANCE HYPOTHESIS}

We have shown that the low proportion of tours with crossings begs for an explanation, not the high proportion of tours that respect the convex hull (see Observation 1). As noted, the convex-hull hypothesis does not explain the low proportion of tours with crossings (see Observation 2). Therefore, we propose the crossingavoidance hypothesis. This hypothesis states that humans aim to avoid crossed lines in the plane when trying to solve E-TSP because they are sensitive to the fact that tours with crossed lines are nonoptimal.

Here we argue that the observation that crossings are nonoptimal is more elementary than the observation that optimal tours follow the convex hull. Consequently, it seems more plausible to assume that people are at least sensitive to the fact that optimal tours have no crossings. We note the following observation.

Observation 3. A tour that contains at least one crossing is nonoptimal (Flood, 1956).

Notice that Observation 3 follows directly from the triangle inequality that holds in the Euclidean plane 


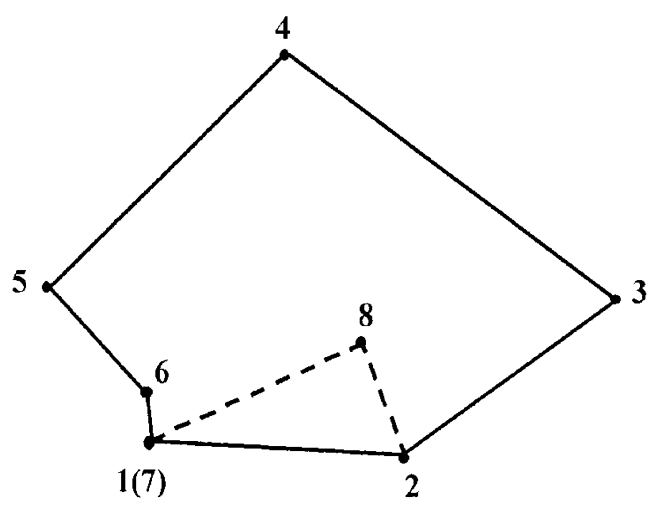

\begin{tabular}{rcc}
\hline & $x$ & $y$ \\
\hline $1(7)$ & 0.300 & 0.282 \\
2 & 0.627 & 0.262 \\
3 & 0.898 & 0.450 \\
4 & 0.473 & 0.746 \\
5 & 0.169 & 0.466 \\
6 & 0.295 & 0.340 \\
8 & 0.572 & 0.400 \\
\hline
\end{tabular}

Figure 2. A point set (left), with $x$ - and $y$-coordinates given for each point (right). The points are numbered in the order in which the heuristic of MacGregor et al. (2000) visits them. Point 1 is the starting point and the direction of travel is counterclockwise. Before Point 8 is inserted, it is closer to the convex-hull edge $(1,2)$ than to any other sketched or connected edge. However, the heuristic will not include Point 8 on the first round, because Point 6 is even closer to the edge $(1,2)$. As a result, the tour has already been closed before Interior Point 8 is inserted. To include Point 8, Point 1 has to be revisited, and the edge $(1,2)$ has to be removed and replaced by the subpath consisting of edges $(1,8)$ and $(8,2)$ (dashed lines). We note that the example uses the smallest number of interior points to demonstrate the problematic behavior of the heuristic. There are instances with more interior points such that this problem even occurs independently of the starting point picked (see MacGregor et al., 2000, for the definition of close as used by their cheapest insertion criterion).

(Figure 3). Simply put, a crossing is nonoptimal because one can always replace the two crossed arcs by two shorter ones. From Observations 1 and 3 we can conclude:

Corollary 1. An optimal tour follows the convex hull (Golden, Bodin, Doyle, \& Stewart, 1980).

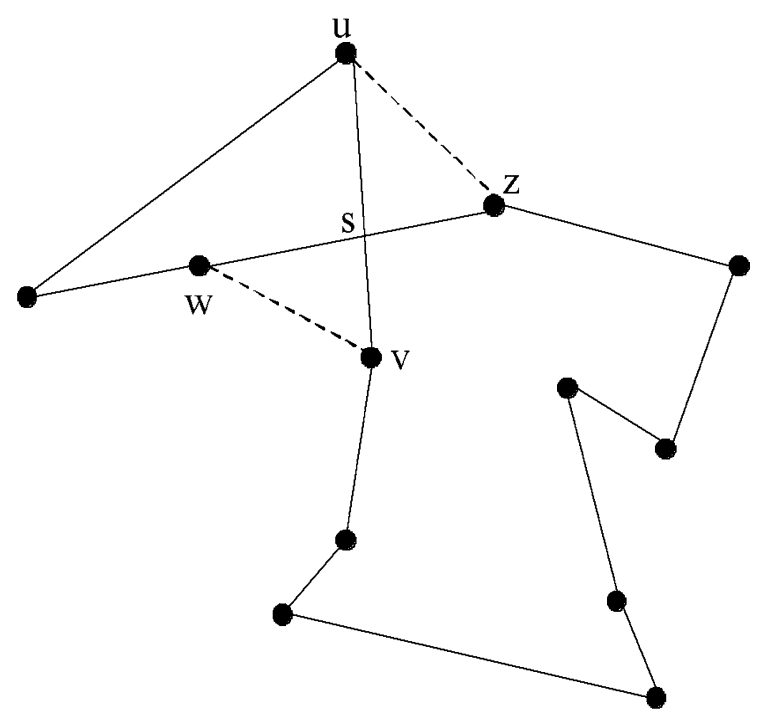

Figure 3. A tour $T$ on some point set $P$ with two edges $(u, v)$ and $(w, z)$ that cross in a point $s \notin P$. We can create a tour $T^{\prime}$ by deleting $(u, v)$ and $(w, z)$ from $T$ and replacing them by $(u, z)$ and $(v, w)$ (dashed lines). Since $d(u, s)+d(s, z)>d(u, z)$ and $d(w, s)+d(s, v)>$ $d(v, w)$, we know that $T^{\prime}$ is shorter than $T$.
We have shown that the property that an optimal tour does not contain any crossings is indeed more elementary than the property that an optimal tour follows the convex hull because the latter derives from the former. MacGregor et al. (2000, p. 1184) stated the issue backwards when they wrote that "the optimal path connects adjacent points on the boundary of the convex hull in sequence ... A corollary is that solutions that do not adhere to this principle will result in crossed arcs, which is clearly nonoptimal." The fact that tours that do not follow the convex hull have crossings is in no sense a corollary of the fact that optimal tours follow the convex hull. Instead, the fact that optimal tours follow the convex hull is implied by Observations 1 and 3. Note that MacGregor et al. (2000) stated explicitly that crossings are clearly nonoptimal (see also Flood, 1956, p. 64). Also Ormerod and Chronicle (1999, p. 1236), who performed experiments in which participants had to judge whether a predrawn tour was optimal or not, considered crossings as indicators of nonoptimality. The nonoptimality of tours with crossings seems intuitive, because it follows directly from a very basic and visually transparent property of the Euclidean plane; that is, the shortest path between two points is a straight line. Therefore it is plausible to assume that people try to avoid crossings when searching for a shortest path or tour.

The crossing-avoidance hypothesis has another advantage: It is more generally applicable than the strategy of following the convex hull. As pointed out by Graham et al. (2000, p. 1197), a convex-hull strategy is potentially useful only in E-TSP, but not in "other spatial problems, such as finding a shortest path between start and a 
goal." Clearly, crossing avoidance is also a good strategy in the latter situation, as well as for problems like minimum Euclidean spanning tree and minimum Euclidean Steiner tree (see, e.g., Garey \& Johnson, 1979, for problem definitions).

We briefly comment on a possible problem for interpretation of the low proportion of tours with crossings that we found when inspecting the raw data from previous experiments. We noticed that some participants produced tours with crossings that were not crossings in a physical sense. That is, a straight line between the two points in question would have produced a crossing, but the participant had chosen to make a detour (i.e., a curved line) so that no physical crossing occurred. The last column in Table 2 lists the frequency of occurrences of such "nonphysical crossings" for each study. The observation of such nonphysical crossings suggests that some participants may misconstrue their task as being the task of finding a tour without lines that cross in the plane. ${ }^{4}$ Such task misconstruals are common dangers in psychological experiments, and the formulation of E-TSP certainly allows for misinterpretation on the participant's side. For example, a participant may (unconsciously) infer that he/she is not supposed to make crossings from the fact that the experimenter did not explicitly say that crossings were allowed. Also, it is possible that for some people the word tour may have the connotation of being a closed path without any crossings. This is not to say that if people would understand their task properly that they would not still tend to avoid crossings (we believe they would). It just draws attention to the importance of making sure that the task is properly understood, to prevent unwarranted attribution of insightfulness on the part of participants.

\section{CONCLUSION}

The convex-hull hypothesis proposes that people aim at following the convex hull when attempting to solve E-TSP instances. One of the main findings presumed to provide support for this hypothesis is that most tours produced by people follow the convex hull (MacGregor \& Ormerod, 1996; MacGregor et al., 1999, 2000). We have shown that this finding does not necessarily provide any evidence for the idea that people aim at following the convex hull; studies have not taken into account the fact that most tours produced by people are crossing free. Namely, a tour that is crossing free by definition follows the convex hull. The only way to show that tours produced by people follow the convex hull because of a reason other than being crossing free is to inspect specifically tours with crossings. We have shown in Table 2 that there is no evidence that such tours tend to follow the convex hull. We also discussed the problematic nature of the other findings that MacGregor and Ormerod (1996) and MacGregor et al. (1999, 2000) claimed are (indirect) support for the convex-hull hypothesis. Contrary to what these authors suggest, we conclude that at the present time there is no evidence for the convex-hull hypothesis.
Finally, we argued that the low proportion of observed tours with crossings invites our crossing-avoidance hypothesis. This hypothesis proposes that people aim at avoiding crossings when attempting to solve E-TSP instances. Unlike the convex-hull hypothesis, the crossingavoidance hypothesis explains the observation that most of the tours produced by people are crossing free. We further argued that this hypothesis is, a priori, at least as plausible as the convex-hull hypothesis.

Of course, the convex-hull hypothesis and crossingavoidance hypothesis are not mutually exclusive. Some preliminary evidence for both the convex-hull hypothesis and the crossing-avoidance hypothesis may be found in verbal reports by participants in the studies by Polivanova (1974) and Vickers et al. (2001). To further test these hypotheses, online monitoring of human strategies as well as verbal protocols may prove more useful.

\section{REFERENCES}

FLood, M. M. (1956). The traveling-salesman problem. Operations Research, 4, 61-75.

GAREY, M. R., \& Johnson, D. S. (1979). Computers and intractability: A guide to the theory of NP-completeness. New York: Freeman.

Golden, B. L., Bodin, L. D., Doyle, T., \& STewart, W. (1980). Approximate traveling salesman algorithms. Operations Research, 28, 694-711.

Graham, S. M., Joshi, A., \& Pizlo, Z (2000). The traveling salesman problem: A hierarchical model. Memory \& Cognition, 28, 1191-1204.

LEE, M. D., \& Vickers, D. (2000). The importance of the convex hull for human performance on the traveling salesman problem: A comment on MacGregor and Ormerod (1996). Perception \& Psychophysics, 62, 226-228.

MacGregor, J. N., \& Ormerod, T. C. (1996). Human performance on the traveling salesman problem. Perception \& Psychophysics, 58, 527-539.

MacGregor, J. N., \& Ormerod, T. C. (2000). Evaluating the importance of the convex hull in solving the Euclidean version of the traveling salesperson problem: Reply to Lee and Vickers (2000). Perception \& Psychophysics, 62, 1501-1503.

MacGregor, J. N., Ormerod, T. C., \& Chronicle, E. P. (1999). Spatial and contextual factors in human performance on the traveling salesperson problem. Perception, 28, 1417-1427.

MacGregor, J. N., Ormerod, T. C., \& Chronicle, E. P. (2000). A model of human performance on the traveling salesperson problem. Memory \& Cognition, 28, 1183-1190.

Ormerod, T. C., \& Chronicle, E. P. (1999). Global perceptual processing in problem solving: The case of the traveling salesperson. Perception \& Psychophysics, 61, 1227-1238.

Polivanova, N. I. (1974). On some functional and structural features of the visual-intuitive components of a problem-solving process. Voprosy Psychologii [Questions in Psychology], 4, 41-51.

Schactman, A. (2002). Children's performance on the traveling salesperson problem. Unpublished honors thesis, University of Victoria, Victoria, BC.

Vickers, D., Butavicius, M., Lee, M. D., \& Medvedev, A. (2001). Human performance on visually presented traveling salesman problems. Psychological Research, 65, 34-45.

\section{NOTES}

1. The word instance is used to refer to a particular instantiation of the E-TSP problem (i.e., a particular set of points for which one is to find a shortest tour), whereas the word problem is used to refer to the generic problem, E-TSP (i.e., given a set of points find a shortest tour).

2. Also revisiting a point, traversing an edge more than once, or a nonphysical crossing was scored as a crossing. 
3. In Schactman (2002) 18 adults were presented with 15 random point sets, with 5,10 , or 15 points. Point sets were presented on paper and participants were instructed to draw the shortest possible tour through the set of points. Performance of the adult group was comparable to that observed in previous studies. That is, mean deviation from optimal (defined as, [observed tour length-optimal tour length] / optimal tour length) was generally low and increased with problem size
( $M=0.016, M=0.020$, and $M=0.027$, for the 5-, 10-, and 15-point instances, respectively).

4. Interestingly, the lowest proportion of tours with crossings (only 1 out of 1,260 tours) was found by Graham et al. (2000) in an experiment in which it was impossible for participants to draw curved lines (i.e., Graham et al. used a computer interface that allowed people to connect points only by straight lines).

(Manuscript received December 4, 2001;

revision accepted for publication October 7, 2002.) 